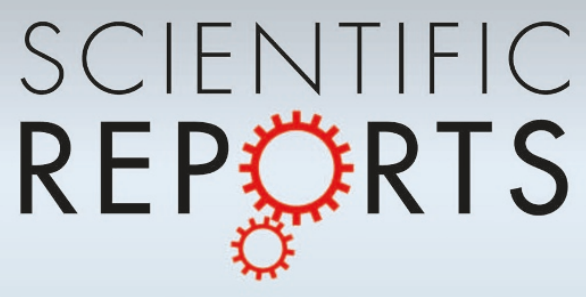

OPEN

SUBJECT AREAS:

PEPTIDES

MOLECULAR EVOLUTION

COMBINATORIAL LIBRARIES

Received

17 February 2014

Accepted

15 July 2014

Published

19 September 2014

Correspondence and requests for materials should be addressed to

P.W. (pinwang@usc. edu) or R.W.R.

(richrob@usc.edu)

\section{Serum Stable Natural Peptides Designed by mRNA Display}

\author{
Shannon M. Howell' ${ }^{\text {, Stephen V. Fiacco' }}$, Terry T. Takahashi ${ }^{1}$, Farzad Jalali-Yazdi ${ }^{2}$, Steven W. Millward ${ }^{3}$, \\ Biliang $\mathrm{Hu}^{2}$, Pin Wang ${ }^{2}$ \& Richard W. Roberts ${ }^{1,2}$
}

'Department of Chemistry, University of Southern California, Los Angeles, California 90089, United States, ${ }^{2}$ Mork Family Department of Chemical Engineering and Materials Science, Viterbi School of Engineering, University of Southern California, Los Angeles CA, 90089-1211, ${ }^{3}$ Department of Cancer Systems Imaging, University of Texas MD Anderson Cancer Center, Houston, TX.

Peptides constructed with the 20 natural amino acids are generally considered to have little therapeutic potential because they are unstable in the presence of proteases and peptidases. However, proteolysis cleavage can be idiosyncratic, and it is possible that natural analogues of functional sequences exist that are highly resistant to cleavage. Here, we explored this idea in the context of peptides that bind to the signaling protein Gail. To do this, we used a two-step in vitro selection process to simultaneously select for protease resistance while retaining function-first by degrading the starting library with protease (chymotrypsin), followed by positive selection for binding via mRNA display. Starting from a pool of functional sequences, these experiments revealed peptides with 100-400 fold increases in protease resistance compared to the parental library. Surprisingly, selection for chymotrypsin resistance also resulted in similarly improved stability in human serum $(\sim 100$ fold $)$. Mechanistically, the decreases in cleavage results from both a lower rate of cleavage $\left(\mathrm{k}_{\mathrm{cat}}\right)$ and a weaker interaction with the protease $\left(\mathrm{K}_{\mathrm{m}}\right)$. Overall, our results demonstrate that the hydrolytic stability of functional, natural peptide sequences can be improved by two orders of magnitude simply by optimizing the primary sequence.

T he major limitation for using peptides as affinity reagents, probes, and therapeutics is their inherent instability in biological environments. Although the amide backbone is chemically stable, peptides are readily broken down in a matter of seconds in the digestive tract, in blood, plasma, serum, and inside cells due to the presence of proteases ${ }^{1}$. Because of this instability, many routes have been devised to chemically alter or modify natural peptides such as 1 ) including the addition of $\mathrm{N}$-methylation to the backbone $\left.{ }^{2}, 2\right)$ insertion of $\beta$ amino acids ${ }^{3,4}, 3$ ) changing the location of the side chain (e.g., peptoids $s^{5,6}$ ), or 4) by covalent cyclization via insertion of chemical bridges ${ }^{7,8}$.

Left unanswered by these studies is the question of how much a functional peptide with natural amino acids could be stabilized by sequence optimization alone. Previously, we demonstrated a route to create high-diversity cyclic peptide libraries via mRNA display ${ }^{9,10}$ and used this approach to isolate a high affinity binder termed cycGiBP to the signaling protein Gail-GDP ${ }^{11}$. cycGiBP showed a very high affinity $\left(\mathrm{K}_{\mathrm{d}}=2.1 \mathrm{nM}\right)$ and $\sim 3$-fold increase in protease resistance as compared with the corresponding linear sequence. One observation in that work was that only one of the three possible cleavage sites predominated when cycGiBP was subjected to chymotrypsin. Using the standard substrate notation for proteases $\left(\mathrm{P}_{3}-\mathrm{P}_{2}-\mathrm{P}_{1}-\mathrm{P}_{1}{ }^{\prime}-\mathrm{P}_{2}{ }^{\prime}-\mathrm{P}_{3}{ }^{\prime} ; \mathrm{P}^{\prime}\right.$ s represent the amino acid identity at a position and the scissile bond is located between $\mathrm{P}_{1}$ and $\mathrm{P}_{1}{ }^{\prime}$ residue), chymotrypsin has a strong $\mathrm{P}_{1}$ preference for $\mathrm{W}>\mathrm{Y}>\mathrm{F} \gg \mathrm{L}^{12}$. However, cycGiBP shows cleavage at $\mathrm{P}_{1}=\mathrm{Y}_{5}$, but not at $\mathrm{P}_{1}=\mathrm{W}_{4}$ or $\mathrm{F}_{7}^{11}$.

We wondered if there were other members in that library that had improved protease resistance while retaining binding function. To address this issue, we performed a dual selection for chymotrypsin resistance and binding function on a library previously only sieved for binding function. Those experiments resulted in a several highly protease resistant peptides and indicate that sequence optimization can improve hydrolytic stability by as much as 400-fold compared to peptides sequences isolated without this selective pressure.

\section{Methods}

E. coli expression of Gail-GDP and mRNA display. Gail-GDP with a C-terminal BirA tag was expressed and purified as previously described $^{13}$. mRNA display selection targeting Gail-GDP was performed starting at round 7 of our previous work and performed as described $^{11}$ with the modification that the cDNA fusions were digested with $2 \mathrm{mg}$ of immobilized chymotrypsin (Sigma-Aldrich) per $10^{6} \mathrm{cpm}$ of fusions at room temperature for 15 minutes in $50 \mathrm{mM}$ Sodium Phosphate buffer $(\mathrm{pH}=8.0)$. The chymotrypsin beads were removed by centrifugation through $0.45 \mu \mathrm{m}$ filters before the selection step. 
Peptide synthesis. R6A (MSQTKRLDDQLYWWEYL), Biotin-labeled R6A (BioMSQTKRLDDQLYWWEYL), cycPRP-1 (MITWIDFISPSK), cycPRP-2 (MTWFEYLSGSK), cycPRP-3 (MTWFEFLSSTSK), and cycGiBP (MITWYEFVAGTK) were synthesized on Rink Amide AM Resin LL (Novabiochem) and cyclized using DSG as described by Millward et $\mathrm{al}^{11}$. After the reaction the cyclized peptides were purified via $\mathrm{C}_{18}$ HPLC and the mass confirmed by MALDITOF MS.

Binding constant determination. Binding constants were determined relative to the R6A peptide $\left[\mathrm{K}_{\mathrm{d}}=60 \mathrm{nM}\right]^{14}$ by equilibrium competition using ${ }^{35}[\mathrm{~S}]$-Met radiolabeled Gail-GDP and biotinylated R6A as previously described ${ }^{10}$ and the data analyzed using GraphPad Prism 5.0.

Protease resistance. Peptides (250 nmol of peptide in DMSO) were added to $50 \mathrm{mM}$ sodium phosphate buffer ( $\mathrm{pH} 8.0$ ) with a final DMSO concentration of $2 \%(\mathrm{v} / \mathrm{v})$. Sixty units of immobilized chymotrypsin (Sigma Aldrich) were added and allowed to incubate at room temperature. Aliquots were taken at various time points and subsequently filtered. The aliquots were then injected onto a $\mathrm{C}_{18}$ reverse phase column and separated by a gradient elution from 15 to $90 \%$ B in 25 minutes. Solvent A consisted of $0.1 \%(\mathrm{v} / \mathrm{v})$ TFA in water and solvent $\mathrm{B}$ contained $\mathrm{CH}_{3} \mathrm{CN}$ with $0.1 \%(\mathrm{v} / \mathrm{v})$ TFA. The area under the starting material peak was quantitated using the 32 KaratGold Software package (Beckman). The graph was generated by fitting the data to a one phase exponential decay equation (GraphPad Prism 5.0). The mean and the standard error are reported in table 1.

$\mathrm{K}_{\mathrm{m}}$ and $\mathrm{V}_{\max }$ determination. The peptides were prepared and characterized as described above in the protease resistance experiment. Only a single 2 minute time point with varying concentrations of the peptides $(0,5,11,22,65$, and $260 \mu \mathrm{M})$ was analyzed using Michaelis-Menten enzyme kinetics regression equation (GraphPad Prism 5.0). The mean and the standard error are reported in table 3.

Human serum digests. Lyophilized human serum (Thermo Scientific) was reconstituted by adding $2 \mathrm{~mL} \mathrm{ddH_{2 }} \mathrm{O}$ to each $5 \mathrm{mg}$ vial from the manufacturer. Each

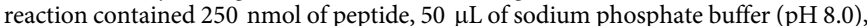
and $10 \%$ DMSO (v/v). One milliliter of reconstituted serum was added to each sample and incubated at $37^{\circ} \mathrm{C}$. For each time point, $100 \mu \mathrm{L}$ aliquots were taken from the reaction and quenched in $300 \mu \mathrm{L}$ of acetonitrile. These quenched samples were centrifuged to separate precipitate, and the supernatant was diluted in water to $1.5 \mathrm{~mL}$. Samples were then analyzed via HPLC as described above. The mean and the standard error are reported in table 3.

Circular dichroism spectroscopy (CD). Far UV-CD spectra were obtained using a Jasco J810 spectropolarimeter (located at the USC NanoBiophysics Core Facility) equipped with a Peltier device. The peptides $(25-100 \mu \mathrm{M})$ were prepared in $10 \mathrm{mM}$ phosphate buffer at $\mathrm{pH} 7.4$ and placed in a $1 \mathrm{~mm}$ path length cuvette. Thereafter, $\mathrm{CD}$ spectra were recorded in the range of 195-240 nm. Five spectra were acquired, and averaged. Spectra were baseline corrected by subtracting blank spectra of the corresponding solutions without peptide and ellipticities were converted to mean residue molar ellipticities in degrees $\mathrm{cm}^{2} \mathrm{dmol}^{-1}$.

\section{Results and Discussion}

Previously, we created a trillion-member mRNA display cyclic peptide library with the form MXXXXXXXXXK (termed $\mathrm{MX}_{10} \mathrm{~K}$ ) and used seven rounds of selection to isolate cyclic peptides that bind to the signaling protein G $\alpha i 1-G D P^{11}$. The best peptide from that selection was cyclic GiBP (cycGiBP), a specific, 12-residue cyclic peptide with a $\mathrm{K}_{\mathrm{d}}=2.1 \mathrm{nM}$. Additionally, we found that cycGiBP was $\sim 3$ fold more resistant to chymotrypsin as compared to a linear version of the peptide ${ }^{11}$.
In an effort to see if protease-resistant, natural-sequence peptides could be found, we took the Pool 7 library from our original experiment (Figure 1a) and subjected it to a two-step selection protocol (Figure 1b). After cyclization with DSG, the library of mRNA peptide fusions was first subjected to degradation by immobilized chymotrypsin for 15 minutes at room temperature and then selected for binding to the target of interest, here Gail-GDP. We chose the 15 minute digestion time because preliminary experiments showed that more than $90 \%$ of the library was degraded under these conditions (data not shown) and our previous results indicated that cycGiBP should have been similarly degraded ${ }^{11}$. We reasoned this incubation should thus provide sufficient selection pressure to reveal proteaseresistant sequences. After three rounds of selection, representative clones from Pool 10 were sequenced (Figure 1c).

We then chose three sequences from pool 10 for further characterization. We term these molecules cycPRPs for Cyclic Protease Resistant Peptides (cycPRP-1, cycPRP-2, and cycPRP-3). The cycPRP sequence consensus in Pool 10 is relatively similar to the consensus sequence seen in Pool 7 and also in other Gail-GDP binding peptides. The core sequence of the cycPRPs (TWIDFI, TWFEYL, TWFEFL) is very similar to cycGiBP (TWYEFV) ${ }^{10}, \mathrm{R} 6 \mathrm{~A}$ $(\text { YWWEYL) })^{14}, \mathrm{~KB}-752$ (TWYDFL) $)^{15}$, the GSP peptide (TVWEFL) ${ }^{13}$, and the AR6-05 peptides (YWWEFL) ${ }^{16}$ selected by both our lab and others. The fact that the sequence consensus is similar suggests that the cycPRP peptides bind to G $\alpha \mathrm{il}-\mathrm{GDP}$ and proteolysis did not destroy binding. Additionally, all previously selected peptides bind near switch 2 of Gail-GDP, and based on this observation, we hypothesize that the cycPRPs bind to the same site on Gail-GDP.

To verify that the cycPRPs bind to G $\alpha$ il-GDP as well as determine binding constants, the cycPRPs were initially assessed by in vitro pull-down with immobilized Gail-GDP. These data indicated that all three molecules were functional, with cycPRP-3 being the best binder followed by cycPRP-1 and cycPRP-2 (data not shown). Consistent with this view, we determined the $\mathrm{K}_{\mathrm{d}}$ values of cycPRP3 to be $9 \pm 2 \mathrm{nM}$ and cycPRP-1 to be $90 \pm 20 \mathrm{nM}$ for Gail-GDP. This binding is excellent compared to KB-752 ( $\left.\mathrm{K}_{\mathrm{d}}=3.9 \mathrm{uM}\right)$, and on par with to cycGiBP $\left(\mathrm{K}_{\mathrm{d}}=2.1 \mathrm{nM}\right)$, raising the possibility that the dual selection optimized the product of protease resistance and binding affinity, rather than one or the other.

The fact that the sequences retained a large fraction of aromatic residues in the core motif was somewhat surprising given the strong preference for chymotrypsin to cleave the backbone adjacent to tryptophan, tyrosine, or phenylalanine $e^{16,17}$. Indeed, our initial prediction was that the chymotrypsin selection would result in sequences lacking aromatic residues that still retained binding function, which clearly did not occur. Based on the primary sequence, cycPRP-1, cycPRP-2 and cycPRP-3, each have 2-3 chymotrypsin cleavage sites (Figure 2), essentially the same as peptides isolated originally without protease challenge ${ }^{11}$. Initial HPLC/MALDI analysis of chymotrypsin-cleaved peptides revealed that only one cut site was predominant a

Pool 7

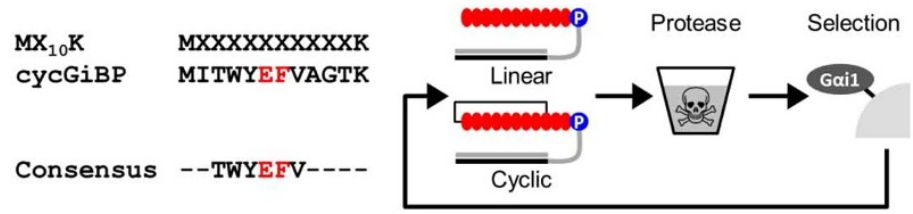

C

\section{Pool 10}

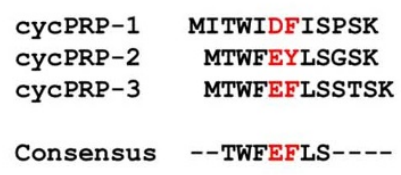

Figure 1 mRNA Display selection for chymotrypsin resistance. (a) Pool 7 of the cyclic peptide library (MX ${ }_{10} \mathrm{~K}$ ) targeting G $\alpha$ i1-GDP was used as the starting point for the selection. (b) In rounds $8-10$, the library was cyclized and subjected to chymotrypsin degradation and binding selection. (c) Representative clones from Pool 10 were sequenced (see supplemental information) and peptides cycPRP-1, cycPRP-2, and cycPRP-3 were further characterized. 


\begin{tabular}{|lcc|} 
Table $1 \mid$ & Chymotrypsin resistance of linear and cyclic \\
Peptide & Linear Half-Life (Minutes) & Cyclic Half-Life (Minutes) \\
\hline GiBP & $0.3 \pm 0.1$ & $2.6 \pm 0.1$ \\
cycPRP-1 & $60 \pm 4$ & $90 \pm 10$ \\
cycPRP-2 & $63 \pm 6$ & $90 \pm 40$ \\
cycPRP-3 & $36 \pm 1$ & $26 \pm 2$ \\
\hline
\end{tabular}

in each of the cycPRP sequences, similar to the result observed originally for cycGiBP. Interestingly, the major cut site varies, depending on the sequence-occurring between Y-E in cycGiBP, W-I in cycPRP-1, and W-F in cycPRP-2 and cycPRP-3 (Figure 2).

We next determined the half-life of the cycPRPs as substrates for chymotrypsin, under conditions similar to the chymotrypsin digestion during the selection (Table 1). The linear GiBP peptide provides a reference point to gauge improvements in protease resistance in this series-and we can use this fixed point to address the effects of protease challenge, cyclization, as well as comparing variations in the primary sequence. Regarding the overall effect of the selection, we observed a 100-200 fold improvement in linear PRPs resistance and a 100-300 fold increase of cycPRPs resistance to chymotrypsin, as compared with linear GiBP. The improved half-life of the PRPs ( $t_{1 / 2}$ $=26$ to $90 \mathrm{~min}$ ) is also consistent with the peptides being able to withstand the 15 minute chymotrypsin digestion largely intact. Regarding cyclization, it is clear that cyclization improves chymotrypsin resistance for cycGiBP, but for the protease resistant peptides, these gains are either quite modest (2-fold for cycPRP-1) or nonexistent (cycPRP-3). The data thus indicate that cyclization is not the primary driver of chymotrypsin resistance in peptides selected using this protease challenge.

There are no obvious variations in the primary sequence that would indicate the cycPRPs have improved chymotrypsin resistance as compared with cycGiBP. Put another way, there is no sequence model of which we are aware that predicts the cycPRPs should be more protease resistant than cycGiBP. Amongst the cycPRPs, the number of aromatic amino acids is almost identical to cycGiBP and there are no systematic alterations in the number of betabranched amino acids, prolines, glycines, or other specific residues near or adjacent to the cut sites. Indeed, at least one statistical model predicts the W-F dipeptide seen at the $\mathrm{P}_{1}-\mathrm{P}_{1}{ }^{\prime}$ position in cycPRP-2 and cycPRP-3 would be particularly susceptible to cleavage ${ }^{16}$. Previous kinetic work with model substrates indicates that $\mathrm{W}$ at $\mathrm{P}_{1}$ is a markedly better substrate than $\mathrm{Y}$ or $\mathrm{F}\left(\operatorname{rank}\right.$ order $\mathrm{P}_{1} ; \mathrm{W}>\mathrm{Y}>$ $F)^{12}$. In this view, cycGiBP could be seen as anomalous, since the primary cleavage site occurs between $\mathrm{Y}$ and $\mathrm{E}$, even though there is a $\mathrm{W}$-Y dipeptide step present.

Our work here differs from model kinetic studies in that each of our substrates contains multiple possible cleavage sites. On the other hand, exhaustive analysis and alignment of many model substrates might be expected to reveal the importance of residues at other positions in the chain to the overall cleavage rate. Schellenberger et $\mathrm{al}^{17}$., constructed exactly such a QSAR model based on available $\mathrm{k}_{\mathrm{cat}} /$ $\mathrm{K}_{\mathrm{m}}$ data and found that $\mathrm{k}_{\mathrm{cat}} / \mathrm{K}_{\mathrm{m}}$ depends on the residue identity from $\mathrm{P}_{3}$ to $\mathrm{P}_{2}{ }^{\prime}$. Including residues outside this window did not improve the predictive power of the model, and the primary determinant at the $\mathrm{P}_{2}{ }^{\prime}$ position was whether the residue was proline or not. While this

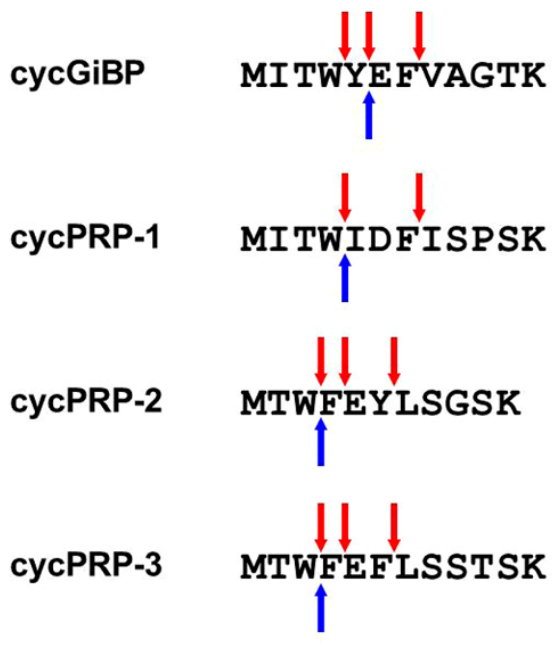

\section{Theoretical Cut Sites Observed Cut Sites}

Figure $2 \mid$ Theoretical (red) and observed (blue) chymotrypsin digest sites of peptides that bind Gail-GDP. cycGIBP is from Pool 7 of the original selection for function only, while the three cycPRP peptides (cycPRP1-3) are from Pool 10 of the protease/binding selection. Red arrows denote theoretical digest sites and blue arrows show the actual digest sited as determined by MALDI-TOF.

model has several caveats (it treats the effect each position as independent and additive), the overall observations are relevant here. Most notable is the predicted variation in cleavage based on residue identity at each position. For $\mathrm{P}_{3}$ and $\mathrm{P}_{2}$, the model predicts variation in $k_{c a t} / K_{m}$ of 40 -fold ( $>>R>G, D>P, K$ ) and 10 -fold (L,V $>P>$ $A>G$ ) respectively. For $P_{1}$, the model predicts a relatively small variation in $\mathrm{Y}, \mathrm{W}$, and $\mathrm{F}$ (2-fold) and for $\mathrm{P}_{1}{ }^{\prime}$ predicts a 20 -fold variation $(F, A>L, V, G)$.

In order to quantitatively compare our peptides with previous model of chymotrypsin substrates, we determined $\mathrm{k}_{\text {cat }} / \mathrm{K}_{\mathrm{m}}$ values for cycGiBP, cycPRP-1, cycPRP-2, cycPRP-3 (Table 2). The $\mathrm{k}_{\text {cat }} /$ $\mathrm{K}_{\mathrm{m}}$ value for cycGiBP is $1.1 \times 10^{5} \mathrm{sec}^{-1} \mathrm{M}^{-1}$ which is in line with model amide bonded substrates ${ }^{17,18}$, supporting the view that cycGiBP acts as normal substrate for the enzyme. Interestingly, the increased protease resistance of the PRP peptides originates from changes in both $\mathrm{k}_{\text {cat }}$ and $\mathrm{K}_{\mathrm{m}}$-with substrates showing 8-16 fold decreases in binding and 8-50 fold decreases in catalytic cleavage rates. cycPRP-2 and cycPRP- 1 have the smallest $\mathrm{k}_{\text {cat }} / \mathrm{K}_{\mathrm{m}}$ values, reduced 420 -fold and 350 -fold as compared to cycGiBP. This reduction is remarkable because it substantially exceeds what would have been expected if all the aromatic residues had been converted to leucine ${ }^{16}$. cycPRP-3 shows a 110 -fold reduction in $\mathrm{k}_{\text {cat }} / \mathrm{K}_{\mathrm{m}}$ compared to cycGiBP, but is also the highest affinity binder. This data also argues that the selection optimized the product of the binding affinity and the reduction in $\mathrm{k}_{\mathrm{cat}} / \mathrm{K}_{\mathrm{m}}$, such that survival represents a compromise between these selected traits.

Previous observations have demonstrated that, at least with proteins, protease resistance can result from differences in protein struc-

\begin{tabular}{|lcccc}
\hline \multicolumn{4}{l}{ Table $2 \mid K_{\mathrm{m}}$ and $V_{\max }$ enhancements for GiBP and PRPs } & \\
Peptide & $\mathrm{K}_{\mathrm{m}}(\mu \mathrm{M})$ & $\mathrm{V}_{\max }\left(\mu M \min ^{-1}\right)$ & $\mathrm{k}_{\text {Cat }} / \mathrm{K}_{\mathrm{m}}\left(\mathrm{M}^{-1} \sec ^{-1}\right)$ & Fold Improvement \\
\hline GiBP & $1.4 \pm 0.3$ & $37 \pm 1$ & $1.1 \times 10^{5}$ & 1 \\
cyCPRP-1 & $11 \pm 3$ & $0.81 \pm 0.06$ & $3.1 \times 10^{2}$ & 350 \\
cyPRP-2 & $22 \pm 5$ & $1.4 \pm 0.1$ & $2.6 \times 10^{2}$ & 420 \\
cycPRP-3 & $18 \pm 4$ & $4.2 \pm 0.3$ & $9.9 \times 10^{2}$ & 110 \\
\hline
\end{tabular}


ture ${ }^{19,20}$. While peptides are generally unstructured in solution, a possible mechanism that could reduce the cleavage rate of the PRPs would be if the peptides had significant levels of secondary structure. In this model, only the unfolded form of the peptide would be available for binding and cleavage by the enzyme. This model would not account for changes in $\mathrm{k}_{\text {cat }}$, but could contribute to changes in $\mathrm{K}_{\mathrm{m}}$ by decreasing the fraction of free peptide available. To test this, we took the CD spectrum of both the linear and cyclic versions of GiBP, cycPRP-1, cycPRP-2, and cycPRP-3 in phosphate buffer at $10^{\circ} \mathrm{C}$ (Figure 3 ) and at $60^{\circ} \mathrm{C}$ (see supplemental information). The reduced temperature was used in order to maximize structure formation, as peptide folding transitions are often quite broad due to their modest enthalpies of formation ${ }^{21,22}$. The largest structure difference between linear and cyclic molecules is seen for cycGiBP (Figure 3a) and very small differences are seen for cycPRP-1, cycPRP-2, and cycPRP-3 (Figure 3b-d). These spectra do not conform simply to established model helix, sheet, or coil spectra ${ }^{23}$. The lack of a minima near $\lambda=222 \mathrm{~nm}$ indicates the peptides are not helical and the overall spectra look most similar to the anti-parallel beta sheet structure ${ }^{23}$. However, the spectra also are similar to unstructured peptides and show little changes upon heating the samples to $60^{\circ} \mathrm{C}$ (see supplemental information). The CD spectra and lack of temperature dependence are most consistent with both the linear and cyclic peptides lacking regular secondary structure, although they do not eliminate non-canonical folded forms.

The lack of peptide structure and the relatively small importance of cyclization imply that the majority of the protease resistance results from sequence effects that alter the efficiency of these peptides as chymotrypsin substrates. This could be because the peptides are optimized to be poor chymotrypsin substrates or because of more

a

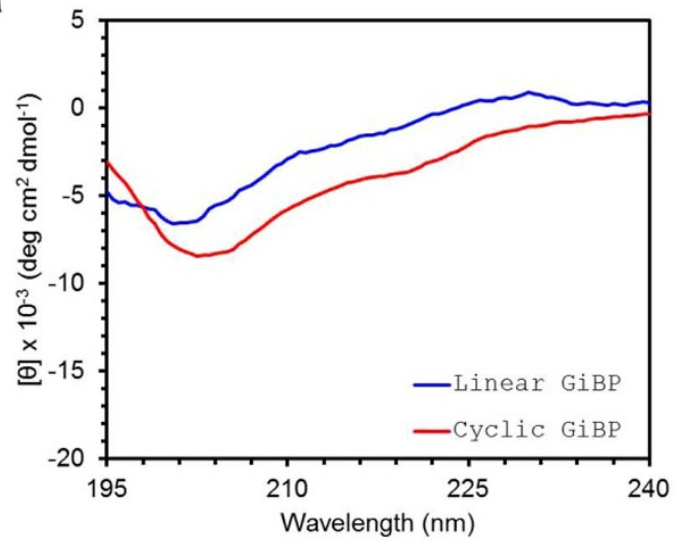

C

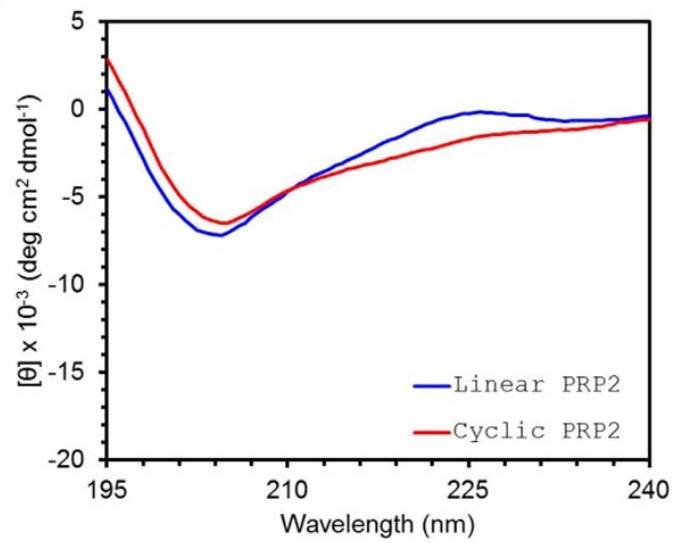

Table 3 | Stability of linear and cyclic peptides in human serum

\begin{tabular}{lcc} 
Peptide & Linear Half-Life (Hours) & Cyclic Half-Life (Hours) \\
\hline GiBP & $<0.02$ & $0.33 \pm 0.02$ \\
PRP-1 & $0.41 \pm 0.02$ & $7.7 \pm 0.4$ \\
PRP-3 & $0.21 \pm 0.02$ & $28 \pm 5$
\end{tabular}

general changes that make the peptides poor protease substrates. To test this, we incubated cycGiBP, cycPRP-1 and cycPRP-3 in human serum containing active proteases and peptidases and examined the half-life of the molecules (Table 3).

Serum generally degrades peptides very quickly ${ }^{24}$ due to the presence of multiple proteases with differing specificity (e.g., thrombin, plasmin, and kallikrein). In line with that view, linear GiBP is undetectable at the first time point after incubation $\left(\mathrm{t}_{1 / 2}<1 \mathrm{~min}\right)$ and cycGiBP shows a half-life of $\sim 20$ minutes. Surprisingly, cyclization of the PRPs gives similar dramatic improvements in stability against serum digestion with increases of 20- to 130-fold for cycPRP-1 and cycPRP-3 respectively. This difference is consistent with serum aminopeptidase, which can digest linear peptides, but whose activity is blocked when the peptides are cyclized ${ }^{25}$. The fact that the cyclic PRPs show dramatically improved stability against digestion by both chymotrypsin and the proteases present in serum, even though the peptides were never exposed to serum proteases during the selection, argues that the peptides have been optimized in a general way to resist proteolytic cleavage. This optimization is somewhat idiosyncratic, as the most stable serum peptide (cycPRP-3) is the least stable of the series against chymotrypsin alone.

b

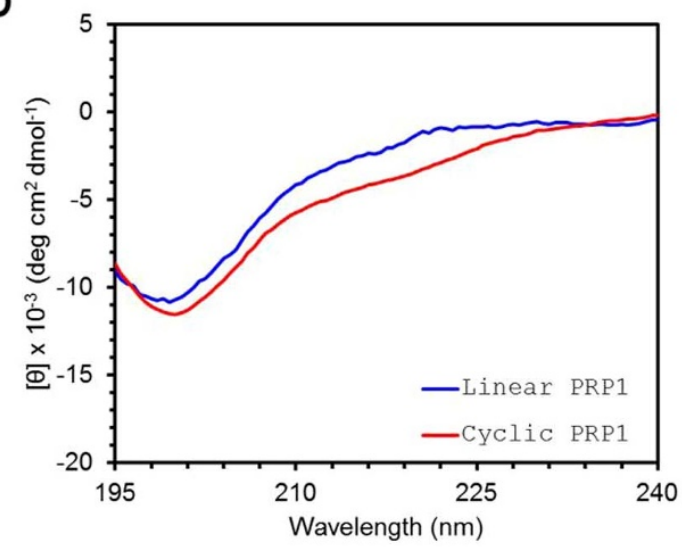

d

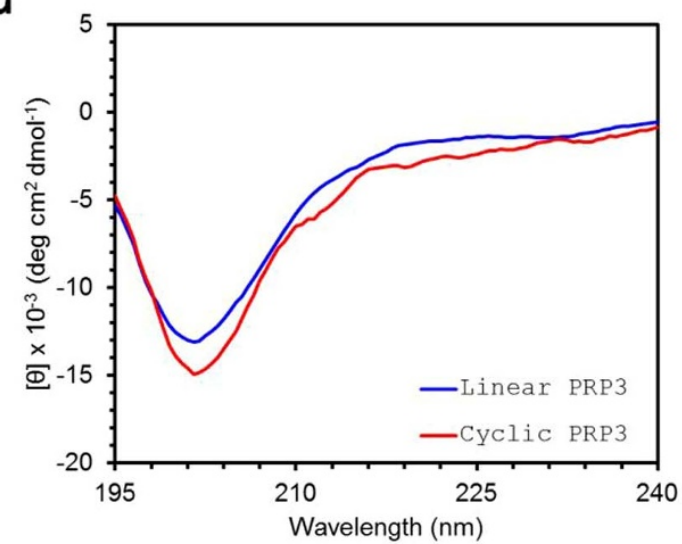

Figure $3 \mid$ CD spectra of linear and cyclic versions of GiBP, PRP-1, PRP-2, and PRP-3. All spectra were taken at $10^{\circ} \mathrm{C}$ in $10 \mathrm{mM}$ phosphate buffer pH 7.4 with linear peptides are shown in blue and cyclic peptides in red. (a) GIBP. (b) PRP-1. (c) PRP-2. (d) PRP-3. 


\section{Conclusions}

Our two-step selection protocol (mRNA display using protease challenge and binding selection) demonstrates that it is possible to isolate highly functional natural peptides that have the ability to bind a target of interest and that are resistant to proteases. This procedure results in stability increases ranging from 100- to 400 -fold. Mechanistically, the protease resistance results from both a lower rate of cleavage $\left(\mathrm{k}_{\mathrm{cat}}\right)$ and a weaker interaction with the enzyme $\left(\mathrm{K}_{\mathrm{m}}\right)$. Surprisingly, the protease resistant peptides do not contain dramatic sequence changes compared with non-protease resistant molecules. Only a few changes are needed to increase proteolytic stability, with 4-6 relatively conservative amino acid changes (out of 10 positions) needed to convert a peptide susceptible to protease into one resistant to protease. These changes are not predictable with existing heuristics/models, do not remove key residues that are substrates for the protease (here W, Y, and F), and do not appear to increase the ordered structure of the molecules. Rather, the changes appear to affect endopeptidase cleavage generally, perhaps through local conformational biases. Nonetheless, these results are exciting because they provide a route to create protease-resistant homologs of biologically-active peptides for use as reagents, diagnostics and therapeutics.

1. McGregor, D. P. Discovering and improving novel peptide therapeutics. Curr. Opin. Pharmacol. 8, 616-619 (2008).

2. Donzel, B., Goodman, M., Rivier, J., Ling, N. \& Vale, W. Synthesis and conformations of hypothalamic hormone releasing factors: two QRF-analogues containing backbone N-methyl groups. Nature 256, 750-751 (1975).

3. Dado, G. P. \& Gellman, S. H. Intramolecular Hydrogen-Bonding in Derivatives of Beta-Alanine and Gamma-Amino Butyric-Acid - Model Studies for the Folding of Unnatural Polypeptide Backbones. J. Am. Chem. Soc. 116, 1054-1062 (1994).

4. Seebach, D. \& Matthews, J. L. beta-peptides: a surprise at every turn. Chem. Commun. 2015-2022 (1997).

5. Owens, R. A., Gesellchen, P. D., Houchins, B. J. \& DiMarchi, R. D. The rapid identification of HIV protease inhibitors through the synthesis and screening of defined peptide mixtures. Biochem. Biophys. Res. Commun. 181, 402-408 (1991).

6. Simon, R. J. et al. Peptoids: A modular approach to drug discovery. Proc. Natl. Acad. Sci. USA 89, 9367-9371 (1992).

7. Blackwell, H. E. et al. Ring-closing metathesis of olefinic peptides: Design, synthesis, and structural characterization of macrocyclic helical peptides. J. Org. Chem. 66, 5291-5301 (2001).

8. Walensky, L. D. et al. Activation of apoptosis in vivo by a hydrocarbon-stapled BH3 helix. Science 305, 1466-1470 (2004).

9. Roberts, R. W. \& Szostak, J. W. RNA-peptide fusions for the in vitro selection of peptides and proteins. Proc. Natl. Acad. Sci. USA 94, 12297-12302 (1997).

10. Millward, S. W., Takahashi, T. T. \& Roberts, R. W. A General Route for PostTranslational Cyclization of mRNA Display Libraries. J. Am. Chem. Soc. 127, 14142-14143 (2005).

11. Millward, S. W., Fiacco, S., Austin, R. J. \& Roberts, R. W. Design of Cyclic Peptides that Bind Protein Surfaces with Antibody-Like Affinity. ACS Chem. Biol. 2, 625-634 (2007).

12. Hedstrom, L., Szilagyi, L. \& Rutter, W. J. Converting trypsin to chymotrypsin: the role of surface loops. Science 255, 1249-1253 (1992).

13. Austin, R. J., Ja, W. W. \& Roberts, R. W. Evolution of class-specific peptides targeting a hot spot of the Gas subunit. J. Mol. Biol. 377, 1406-1418 (2008).

14. Ja, W. W. \& Roberts, R. W. In vitro selection of state-specific peptide modulators of G protein signaling using mRNA display. Biochemistry 43, 9265-9275 (2004).
15. Johnston, C. A. et al. Structure of Galpha(i1) bound to a GDP-selective peptide provides insight into guanine nucleotide exchange. Structure (Camb) 13, 1069-1080 (2005).

16. Folk, J. E. \& Cole, P. W. Chymotrypsin C. Ii. Enzymatic Specificity toward Several Polypeptides. J. Biol. Chem. 240, 193-197 (1965).

17. Schellenberger, V., Braune, K., Hofmann, H. J. \& Jakubke, H. D. The specificity of chymotrypsin. A statistical analysis of hydrolysis data. Eur. J. Biochem./FEBS 199, 623-636 (1991).

18. Hedstrom, L. Serine protease mechanism and specificity. Chem. Rev. 102, 4501-4524 (2002).

19. Fung, B. K. \& Nash, C. R. Characterization of transducin from bovine retinal rod outer segments. II. Evidence for distinct binding sites and conformational changes revealed by limited proteolysis with trypsin. J. Biol. Chem. 258, 10503-10510 (1983).

20. Hurley, J. B., Simon, M. I., Teplow, D. B., Robishaw, J. D. \& Gilman, A. G. Homologies between signal transducing $G$ proteins and ras gene products. Science 226, 860-862 (1984).

21. Marqusee, S. \& Baldwin, R. L. Helix stabilization by Glu $-\ldots$..Lys + salt bridges in short peptides of de novo design. P. Natl. Acad. Sci. USA 84, 8898-8902 (1987).

22. Marqusee, S., Robbins, V. H. \& Baldwin, R. L. Unusually stable helix formation in short alanine-based peptides. P. Natl. Acad. Sci. USA 86, 5286-5290 (1989).

23. Compton, L. A. \& Johnson, W. C., Jr. Analysis of protein circular dichroism spectra for secondary structure using a simple matrix multiplication. Anal. Biochem. 155, 155-167 (1986).

24. Powell, M. F. et al. Peptide stability in drug development. II. Effect of single amino acid substitution and glycosylation on peptide reactivity in human serum. Pharm. Res. 10, 1268-1273 (1993).

25. Wildes, D. \& Wells, J. A. Sampling the N-terminal proteome of human blood. P. Natl. Acad. Sci. USA 107, 4561-4566 (2010).

\section{Acknowledgments}

$\mathrm{CD}$ results were obtained using equipment provided by the USC NanoBiophysics Core Facility. This work was supported by NIH GM R01 60416 (R.W.R.), NIH 5R01CA170820 (R.W.R. \& P.W.), NIH 5R01AI085583, and the USC WiSE Merit Fellowship for Doctorate Students (S.M.H.).

\section{Author contributions}

S.M.H. and R.W.R. wrote the main manuscript text. S.V.F., T.T.T. and R.W.R., planned experiments. S.M.H., S.V.F. and F.J., performed the experiments and analyzed the data. R.W.R., S.M.H. and F.J. prepared the figures. S.W.M. provided the starting DNA library. All authors (S.M.H., S.V.F., T.T.T., F.J., S.W.M., B.H., P.W. and R.W.R.) reviewed the manuscript and have given approval to the final version of the manuscript.

\section{Additional information}

Supplementary information accompanies this paper at http://www.nature.com/ scientificreports

Competing financial interests: The authors declare no competing financial interests. How to cite this article: Howell, S.M. et al. Serum Stable Natural Peptides Designed by mRNA Display. Sci. Rep. 4, 6008; DOI:10.1038/srep06008 (2014).

This work is licensed under a Creative Commons Attribution-NonCommercialShareAlike 4.0 International License. The images or other third party material in this article are included in the article's Creative Commons license, unless indicated otherwise in the credit line; if the material is not included under the Creative Commons license, users will need to obtain permission from the license holder in order to reproduce the material. To view a copy of this license, visit http:// creativecommons.org/licenses/by-nc-sa/4.0/ 